\title{
Refinement of intraperitoneal injection of sodium pentobarbital for euthanasia in laboratory rats (Rattus norvegicus)
}

\author{
Katie K Zatroch ${ }^{1}$, Cameron G. Knight ${ }^{2}$, Julie N. Reimer ${ }^{2}$ and Daniel S. J. Pang ${ }^{3,4}$
}

\begin{abstract}
Background: The Canadian Council on Animal Care and American Veterinary Medical Association classify intraperitoneal (IP) pentobarbital as an acceptable euthanasia method in rats. However, national guidelines do not exist for a recommended dose or volume and IP euthanasia has been described as unreliable, with misinjections leading to variable success in ensuring a timely death. The aims of this study were to assess and improve efficacy and consistency of IP euthanasia.

In a randomized, blinded study, 51 adult female Sprague-Dawley rats (170-495 g) received one of four treatments: low-dose low-volume (LL) IP pentobarbital ( $n=13,200 \mathrm{mg} / \mathrm{kg}$ pentobarbital), low-dose high-volume (LH) IP pentobarbital ( $n=14,200 \mathrm{mg} / \mathrm{kg}$ diluted 1:3 with phosphate buffered saline), high-dose high-volume (HH, $n=14$, $800 \mathrm{mg} / \mathrm{kg}$ pentobarbital), or saline. Times to loss of righting reflex (LORR) and cessation of heartbeat (CHB) were recorded. To identify misinjections, necropsy examinations were performed on all rats. Video recordings of $L L$ and $\mathrm{HH}$ groups were analyzed for pain-associated behaviors. Between-group comparisons were performed with 1-way ANOVA and Games-Howell post hoc tests. Variability in CHB was assessed by calculating the coefficient of variation (CV).

Results: The fastest euthanasia method (CHB) was HH (283.7 $\pm 38.0 \mathrm{~s})$, compared with LL (485.8 $\pm 140.7 \mathrm{~s}, p=0.002)$ and $\mathrm{LH}(347.7 \pm 72.0 \mathrm{~s}, p=0.039)$. Values for CV were: HH, 13.4\%; LH, 20.7\%; LL, 29.0\%. LORR time was longest in LL (139.5 $\pm 29.6 \mathrm{~s})$, compared with HH (111.6 $\pm 19.7 \mathrm{~s}, p=0.046)$ and LH (104.2 $\pm 19.3 \mathrm{~s}, p=0.01)$. Misinjections occurred in $17.0 \%$ (7/41) of euthanasia attempts. Pain-associated behavior incidence ranged from $36 \%(4 / 11, L L)$ to $46 \%(5 / 11, H H)$.

Conclusions: These data illustrate refinement of the IP pentobarbital euthanasia technique. Both dose and volume contribute to speed of death, with a dose of $800 \mathrm{mg} / \mathrm{kg}(\mathrm{HH})$ being the most effective method. An increase in volume alone does not significantly reduce variability. The proportion of misinjections was similar to that of previous studies.
\end{abstract}

Keywords: CCAC, Pentobarbital, Killing, Refinement, Welfare

\section{Background}

Over 2 million rats are used in biomedical research in Canada and the European Union annually [1,2]. The overwhelming majority of laboratory studies employing rodents end with killing the animals upon completion of the study or if a humane endpoint has been reached. While this is a reality of research, efforts to refine killing methods, to achieve "euthanasia", for rats and other laboratory animals are ongoing, as reflected in recent

\footnotetext{
*Correspondence: cgknight@ucalgary.ca

${ }^{2}$ Veterinary Clinical and Diagnostic Sciences, Faculty of Veterinary Medicine,

University of Calgary, 3280 Hospital Dr NW, T2N 4 Z6 Alberta, Canada

Full list of author information is available at the end of the article
}

updates to the Canadian Council on Animal Care (CCAC) and American Veterinary Medical Association (AVMA) euthanasia guidelines [3, 4]. Goals for successful euthanasia include techniques requiring minimal restraint, simplicity of administration, and a swift, painless death $[5,6]$.

A commonly employed technique for euthanasia of laboratory rats is an overdose of carbon dioxide. However, current behavioral and physiologic evidence suggests that this method is aversive and may be painful [7-16]. As a result, the CCAC and AVMA have reclassified killing with carbon dioxide as "conditionally acceptable" [4] and "acceptable with conditions" [3]. 
In contrast, an acceptable method and preferred alternative to carbon dioxide is overdose with a barbiturate such as sodium pentobarbital (PB). An intraperitoneal (IP) route of injection is acceptable when intravenous injection cannot be performed or is impractical [3, 4]. Current guidelines do not indicate a specific dose of sodium pentobarbital for euthanasia, although $200 \mathrm{mg} / \mathrm{kg}$ or 3 times the anesthetic dose has been suggested [5]. There are several potential drawbacks associated with IP PB injection, including misinjection, variability in effect and pain [8, 17-23].

An important factor contributing to variability of drug effect (speed of onset and success) is misinjection, with deposition of injectate into intra-abdominal fat, abdominal viscera or the subcutaneous space. In the case of IP pentobarbital for euthanasia this results in a delayed time to death or even failure to cause loss of consciousness. Attempts to reduce variability with a two-person injection technique (one to restrain, one to inject) have had variable success, with reported proportions of misinjections ranging from 6 to $20 \%$ [19-21].

Pain, inferred from behavioral observations, necropsy findings and biomarkers, has also been cited as a potential impediment to achieving the principle of euthanasia. Specifically, exhibition of writhing (defined as the contraction of the abdomen and extension of the hind legs), grossly visible inflammation of abdominal viscera at necropsy and a measurable increase in spinal cord cFos have been reported following IP injection of pentobarbital [17, 18, 22, 23].

The primary aim of this study was to assess the impact of varying the dose and volume of sodium pentobarbital injected into the intraperitoneal cavity on time to death and consistency of the killing process. Secondary aims were identification of misinjections by necropsy and the quantification of writhing behavior in response to IP PB. We hypothesized that speed and consistency of IP euthanasia would be improved by using a higher dose and higher volume.

\section{Methods}

\section{Study design}

Fifty-one adult female Sprague-Dawley rats (170-495 g), sourced as surplus breeding stock, were included in the study. A sample size of approximately 13 animals, to achieve $80 \%$ power with an alpha of 0.05 (with an anticipated $20 \%$ misinjection rate) with an effect size of 1.5, was determined from pilot data. All animals remained in paired housing until the time of trial and were not handled prior to the study. Housing consisted of standard micro-filter cages $(47 \times 25 \times 21 \mathrm{~cm})$ with wood shavings and shredded paper bedding and a plastic tube for enrichment. A 12-12 h lights on-off cycle (lights on at 0700) was maintained in an environmentally controlled room $\left(23{ }^{\circ} \mathrm{C}, 22 \%\right.$ humidity). All experiments were performed during the light period (0730-1800).

Animals were randomly assigned to one of four treatment groups for IP injection. A low-dose low-volume group (LL, $n=13$ ) received $200 \mathrm{mg} / \mathrm{kg}$ sodium pentobarbital (Euthanyl, $240 \mathrm{mg} / \mathrm{ml}$, Bimeda-MTC Animal Health Inc., Cambridge, ON, Canada). A low-dose high-volume group ( $\mathrm{LH}, n=14$ ) received $200 \mathrm{mg} / \mathrm{kg}$ sodium pentobarbital diluted 1:3 with phosphate-buffered saline (PBS). A high-dose high-volume group ( $\mathrm{HH}, n=14)$ received 800 $\mathrm{mg} / \mathrm{kg}$ sodium pentobarbital. A control group $(n=10)$ received $1 \mathrm{ml}$ of PBS. Each treatment was placed in a $1 \mathrm{ml}$ (LL and control groups) or $3 \mathrm{ml}$ ( $\mathrm{LH}$ and $\mathrm{HH}$ groups) syringe as dictated by the volume of injectate. A new $25 \mathrm{G}$ 5/8" hypodermic needle was attached to each syringe for injection. Blue food coloring $(0.01 \mathrm{~mL}$, Club House, Burlington, Ontario) was added to each treatment to facilitate visualization of injectate during necropsy examination.

At the beginning of each trial, a single rat was removed from the housing unit and placed in a Plexiglas chamber $(\mathrm{L} \times \mathrm{W} \times \mathrm{H}: 27.5 \times 14.5 \times 20.5 \mathrm{~cm})$. Two video cameras (Panasonic HC-V720P/PC, Panasonic Canada Inc., Mississauga, ON, Canada) were placed along the long and short axes of the chamber. Before injection, baseline video of the rat was recorded for $10 \mathrm{~min}$. Treatments were prepared in a separate room during baseline video recording. Individuals performing the IP injections and behavioral analyses were blinded to treatment.

Following baseline video, each rat was removed from the box and restrained for a two-person injection technique. Rats were held in dorsal recumbency at an approximately $30^{\circ}$ angle (head lowermost). The holder (DP) supported each rat and restrained the left pelvic limb. The individual administering each injection (KZ) restrained each rat's right pelvic limb, injecting with the right (dominant) hand (Fig. 1).

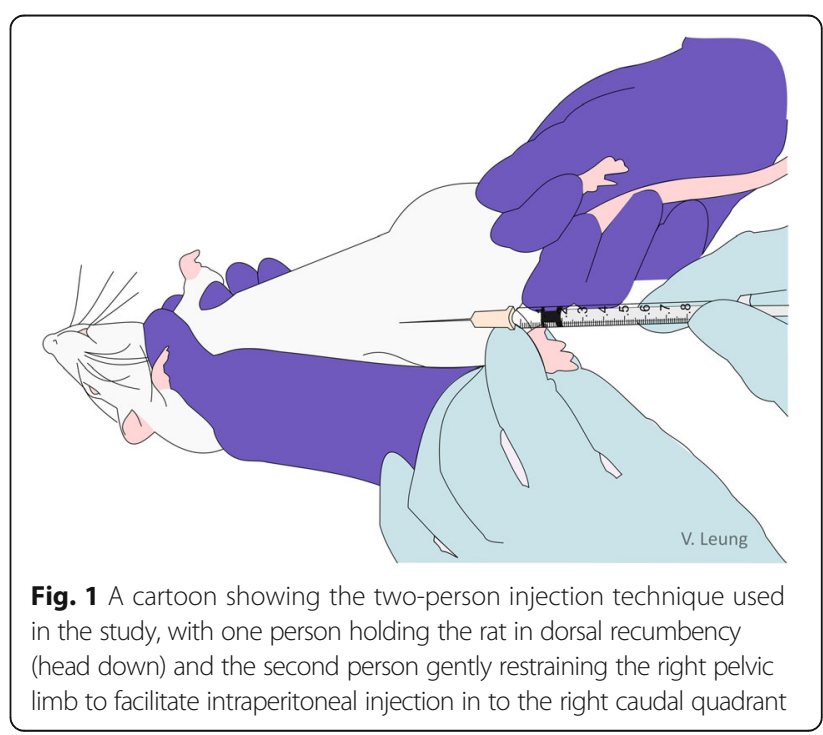


Each injection was performed in the right caudal quadrant of the abdomen at the level of the coxofemoral joint and approximately $5 \mathrm{~mm}$ to the right of midline. The needle was directed cranially at a $45^{\circ}$ angle to the body wall.

Immediately following completion of injection, each rat was returned to the observation chamber. A single blinded observer $(\mathrm{KZ})$ monitored for signs of ataxia (stumbling, falling, crossing feet) following injection. If signs of ataxia were noted, an attempt was made to place the rat in dorsal recumbency to evaluate for a loss of righting reflex (LORR), a surrogate for loss of consciousness [8, 24]. LORR was confirmed if the rat remained in dorsal recumbency for ten seconds. Failure of LORR was established if the rat resisted initial placement on its back or was able to right itself within ten seconds. In cases of initial LORR failure, the test was repeated every $30 \mathrm{~s}$ until LORR occurred. Following LORR, the animal was monitored for onset of apnea, defined as the animal's chest ceasing to rise and fall. If and when apnea occurred, the rat was placed in left lateral recumbency. The left thoracic wall was then auscultated continuously with a stethoscope to identify cessation of heartbeat $(\mathrm{CHB})$. Following $\mathrm{CHB}$ confirmation, video recording was stopped. The observation chamber was cleaned between trials.

When $\mathrm{CHB}$ did not occur within 20 min of IP injection, animals were euthanized with an overdose of carbon dioxide gas using a gradual fill (30\% chamber volume per minute) technique. These cases were considered unsuccessful euthanasias.

\section{Necropsy examination}

Following $\mathrm{CHB}$, each animal was carefully removed from the chamber and positioned in dorsal recumbency for necropsy examination. The skin was incised along the midline and the injection site was identified in the abdominal wall musculature. The abdominal wall was incised and the intestines were reflected out of the abdominal cavity. Distribution of blue injectate and any misinjection into hollow viscera were noted. The liver was reflected cranially and any presence of dye within the biliary vessels caused by uptake of injectate from the peritoneal cavity and subsequent biliary excretion was noted. The GIT from the cardia to the descending colon was removed and any intestinal segments with dye-stained serosa were opened to confirm or rule out intraluminal misinjection. Misinjection was defined as the presence of blue injectate within hollow viscera or subcutaneous tissues, or staining the fur. For each rat, the serosal surfaces of the abdominal wall injection site, the caudate liver lobe, and transverse sections of at least three intestinal sections were examined histologically after formalin fixation for evidence of acute inflammation or swelling of mesothelial cells. Evaluation was performed by a single board-certified veterinary pathologist (CK), who was blinded to treatment group assignments.

\section{Off-line video analysis}

Videos of the $\mathrm{HH}$ and LL trials were analyzed for the incidence of writhing behavior by a single individual blinded to treatment (JR). Baseline recordings were analyzed in their entirety while post-injection videos were analyzed until the rat became ataxic. Videos from the saline group were analyzed post hoc, with a viewing duration of $199 \mathrm{~s}$; the mean time + 2SD for LORR in the LL group. As blinding was limited (two additional PB videos, one pre- and one post-injection, were added to introduce uncertainty), these videos were scored by two observers independently (JR, DP). Writhing was defined as a contraction of the lateral abdominal walls to the extent where the abdomen became concave with concurrent extension of the pelvic limbs $[18,23]$.

\section{Statistical methods}

All statistical analyses were performed using commercial software (GraphPad Prism v.6.03, GraphPad Software, Inc. La Jolla, California, USA and IBM SPSS Statistics 21, IBM, Armonk, NY, USA). Data were considered approximately normal if skewness and kurtosis were less than \pm 1.5 and 3 , respectively. Between-group comparisons were performed with a one-way ANOVA with a Games-Howell multiple comparisons test. Consistency of the euthanasia process was assessed with a coefficient of variation $(\mathrm{CV})$ calculation. Differences in median body weight between those animals in which a misinjection occurred and those with a successful injection were compared with a Mann-Whitney test. A $p$-value of $<0.05$ was considered significant. Data are presented as mean \pm SD.

\section{Results}

Of 51 trials, 43 (84.3\%) were successful IP injections and $8(15.7 \%)$ were misinjections. Successful IP injections were distributed as follows: $\mathrm{PB} ; n=34$ and control; $n=9$. All successful injections with $\mathrm{PB}$ resulted in death: LL $(n=11)$, LH $(n=12)$, and HH $(n=11)$.

The fastest killing method from injection to $\mathrm{CHB}$ was the $\mathrm{HH}$ group $(283.7 \pm 38.0 \mathrm{~s})$, which was significantly faster than both the LL $(485.8 \pm 140.7 \mathrm{~s}, p=0.002)$ and LH (347.7 $\pm 72.0 \mathrm{~s}, p=0.039)$ groups (Fig. 2). Euthanasia in the LH group was also significantly faster than the LL group $(p=0.027)$.

The $\mathrm{HH}$ group was not only the fastest, but also the most consistent euthanasia method. The $\mathrm{CV}$ for $\mathrm{HH}$ was $13.4 \%$, compared with $29.0 \%$ for LL and $20.7 \%$ for LH groups.

The period from injection to LORR was longest in LL $(139.5 \pm 29.6 \mathrm{~s})$, compared with both $\mathrm{HH}(111.6 \pm 19.7$ s, $p=0.046)$ and LH $(104.2 \pm 19.3 \mathrm{~s}, p=0.01$, Fig. 3a). 


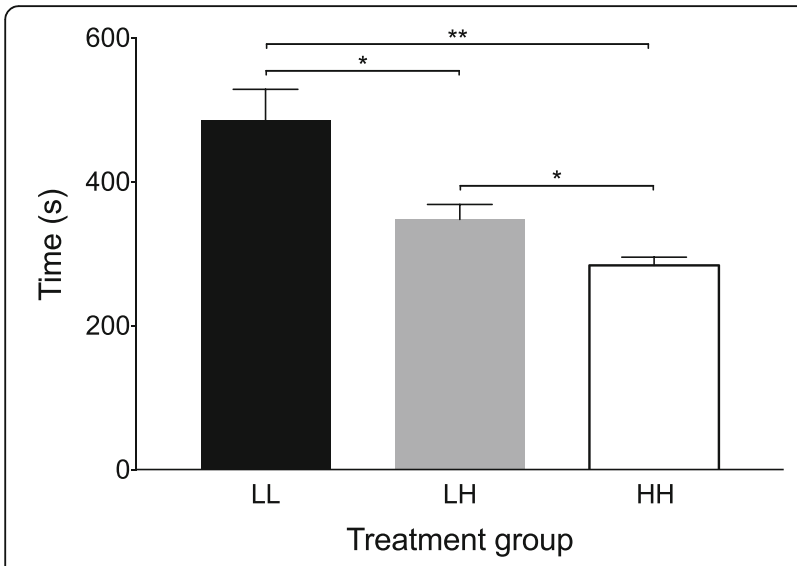

Fig. 2 Time from delivery of the intraperitoneal injection to cessation of heart beat was fastest in the high-dose high-volume group $(\mathrm{HH})$ $\mathrm{LL}=$ low-dose low-volume group, $\mathrm{LH}=$ low-dose high-volume group. ${ }^{*} p<0.05{ }^{* *} p=0.002$
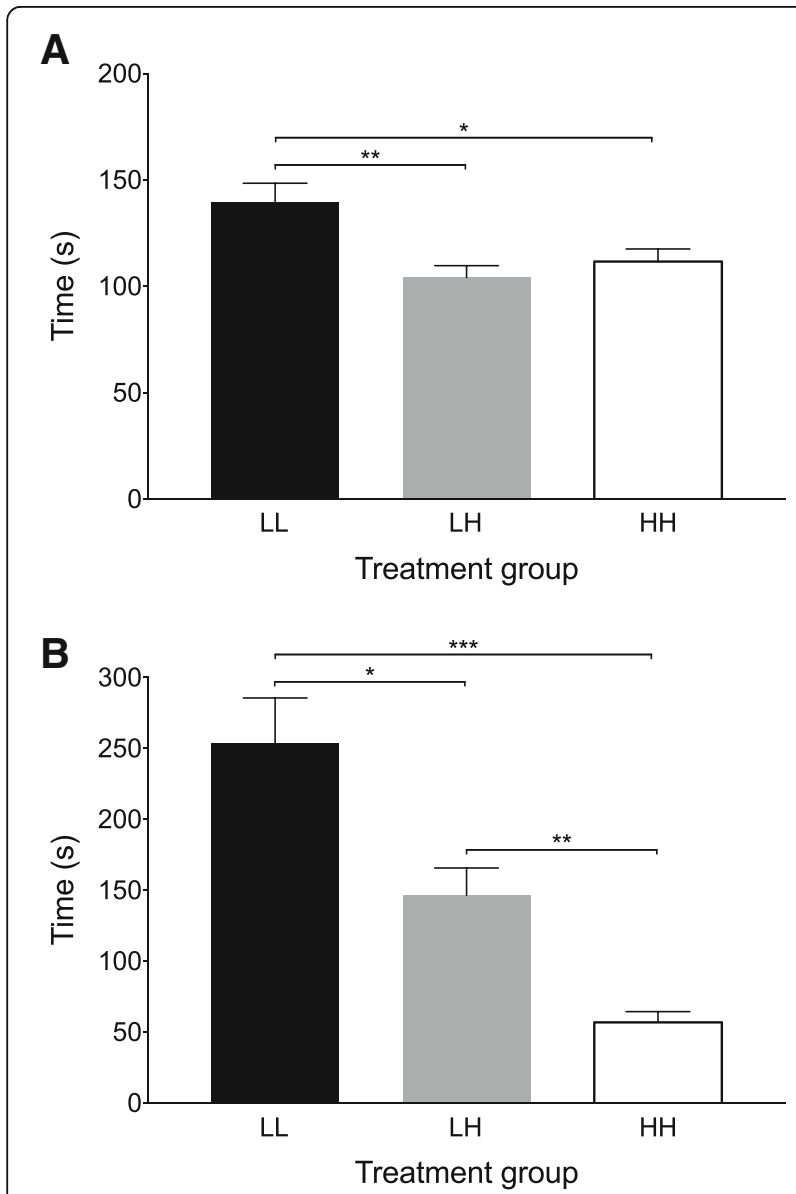

Fig. 3 a Time from delivery of the intraperitoneal injection to loss of the righting reflex was longest in the low-dose low-volume group (LL). $\mathrm{LH}=$ low-dose high-volume group, $\mathrm{HH}=$ high-dose high-volume group. ${ }^{*} p<0.05{ }^{* *} p=0.01$. $\mathbf{b}$ Time from loss of the righting reflex to apnea was shortest in the high-dose high-volume group $(\mathrm{HH}) .{ }^{*} p<0.05{ }^{* *} p=0.002$ ${ }^{* * *} p<0.001$
Time from injection to LORR did not differ between $\mathrm{LH}$ and $\mathrm{HH}(p=0.64)$. In no cases where LORR was confirmed were rats able to right themselves after $10 \mathrm{~s}$ in dorsal recumbency had elapsed. The LORR-apnea time period showed the greatest variation between treatment groups and therefore had the greatest influence on the speed of the overall time to death (Fig. 3b). LORR-apnea was significantly faster in the $\mathrm{HH}$ group $(56.8 \pm 25.1 \mathrm{~s})$ than LL $(253.3 \pm 106.7 \mathrm{~s}, p<0.001)$ and LH $(146.6 \pm$ $66.1 \mathrm{~s}, p=0.002)$. LORR-apnea in the LH group was also significantly faster than in the LL group $(p=0.03)$. There was no significant difference from apnea-CHB among treatment groups: $\mathrm{HH}(116.2 \pm 19.7 \mathrm{~s})$ versus $\mathrm{LH}$ $(93.0 \pm 29.0 \mathrm{~s}, p=0.09), \mathrm{HH}$ versus LL $(92.9 \pm 24.2 \mathrm{~s}$, $p=0.06)$, LH versus LL $(p=1.0)$.

Eight misinjections were identified at necropsy. One misinjection was in a control animal. Seven misinjections were treatment group rats $(\mathrm{HH} ; n=3, \mathrm{LH} ; n=2, \mathrm{LL} ; n=2)$. Of these, euthanasia was unsuccessful (exceeding $20 \mathrm{~min}$ ) in $3(42.8 \%)$ animals ( $\mathrm{HH}[n=2]$, LL $[n=1])$. In the four animals in which euthanasia was successful $(\mathrm{HH}$ $[n=1]$, LH $[n=2]$, LL $[n=1])$, injection-CHB ranged from 318-1200s. There was no difference in body weight between animals with successful injections (325 [170-495]g) or misinjections (363 [285-461]g, $p=0.27,95 \%$ confidence interval of the median difference; -24 to 90 ).

The anatomic distribution of the eight misinjections was as follows: four entered the cecal lumen, two entered the jejunal lumen, one was entirely within the subcutaneous tissues of the abdominal wall, and one was predominantly over the fur of the medial thigh, with a small amount in the subcutaneous space (Fig. 4). Cecal positions were variable: $14 / 51(27.5 \%)$ in the right caudal quadrant, 5/51 (9.8\%) located in the midline and 32/51 $(62.7 \%)$ in the left caudal quadrant.

\section{Writhing}

Writhing was not observed in the saline, LL or $\mathrm{HH}$ groups in baseline video recordings. Following injection, writhing, assessed in animals with successful injections, was seen in $45.5 \%(5 / 11)$ of $\mathrm{HH}$ and $36.4 \%(4 / 11)$ of LL rats. Two animals showed writhing behavior after saline injection $(2 / 9,22 \%)$.

\section{Discussion}

Historically, concerns regarding IP euthanasia have revolved around misinjection leading to variable success rates and the potential for pain $[8,17-21,23]$.

Our results show that: 1 . IP injection with $800 \mathrm{mg} / \mathrm{kg}$ sodium pentobarbital ( $\mathrm{HH}$ group) resulted in the fastest and most consistent killing method; 2 . variable cecal position contributed to misinjections; and 3. the incidence of 

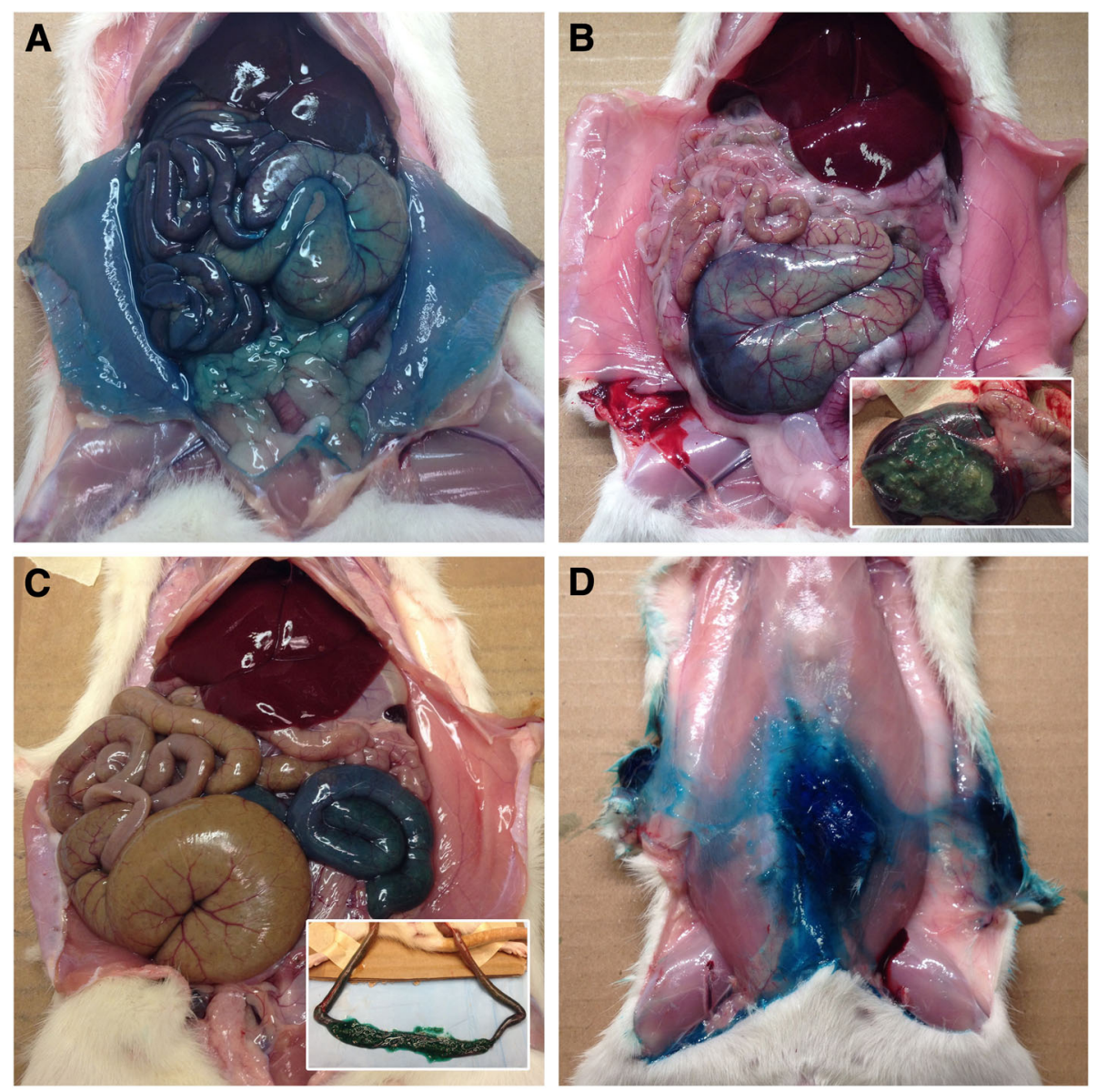

Fig. 4 Abdominal cavities of four rats after confirmation of death; ventral view. Diffuse blue dye staining of serosal surfaces following successful intraperitoneal injection (a). Restricted dye distribution following inadvertent cecal (b), intestinal (c), and subcutaneous (d) misinjection. The insets in panels B and $C$ show dye-stained ingesta, confirming inadvertent luminal misinjection

writhing behavior was less than half of that previously reported.

Both dose and volume contribute to the speed of euthanasia, and dose in particular appears to have the most dramatic effect on consistency of technique. The speed and consistency of the killing process can be improved through an increase in dose (accompanied by an increase in volume). Increasing injectate volume without increasing dose (LH group) improved the speed of IP euthanasia. However, further improvements in speed and consistency were achieved in the $\mathrm{HH}$ group.

From these results, several conclusions can be drawn. The mean + 2SD for the period from completion of injection to LORR was $151.0 \mathrm{~s}$ when $800 \mathrm{mg} / \mathrm{kg}$ pentobarbital (HH group) was administered IP. Therefore, it is highly likely that an animal that maintains LORR beyond this time has experienced a misinjection. If using the period from completion of injection to apnea as the indicator of successful injection, the time for mean +2 SD was $259.1 \mathrm{~s}$. Should these times be exceeded, a second injection of pentobarbital or alternative killing method should be performed.

Any increase in pentobarbital use is associated with an increased cost. For the formulation used here, this equates to approximately US $\$ 0.13$ for the $\mathrm{HH}$ technique in a $250 \mathrm{~g}$ rat. While cost is an important consideration, it should be weighed against the labor cost of the slower (approximately 1.7 fold) LL group and potentially prolonged pain experience during the period from injection to LORR.

Misinjection is a consistent limitation of IP PB. The rate of misinjection in this study was consistent with the range reported in the literature (for injections given in to the caudal right abdominal quadrant), from 6 to $20 \%$ [19-21]. A factor contributing to the misinjection rate is variability in cecal position. IP injection is usually performed in the right caudal abdominal quadrant and previous work has confirmed that the cecum is most commonly located in the left caudal abdominal quadrant (61.9\%, right $24.2 \%$, middle $13.8 \%$, total $n=289$ adult 
male and female rats) [20]. Our results are similar to these findings despite using a different injection technique. In the study of Coria-Avila et al. [20], rats were restrained by a single person and suspended vertically by the thorax with the head up. This suggests that body position during injection has a minimal effect on the incidence of misinjection. Based on the misinjection rates in this and other studies, as well as the positional variation of abdominal viscera noted on necropsy, variations in IP injection methodology are unlikely to eliminate the possibility of misinjections. The wide range in body mass of the animals did not affect the incidence of misinjections, though statistical power to detect the small difference in body weights between treatment groups was low (approximately 20\%). The majority (6/8) misinjections resulted from penetration of the cecal or jejunal lumen, suggesting that the presence of intraabdominal fat did not hinder IP penetration.

Given this inherent obstacle in refining the euthanasia process, we hope that the recommendations described above will facilitate early identification of a misinjection, guiding the decision to repeat the injection or select an alternative euthanasia method.

We observed a substantially lower incidence of writhing behavior than previously reported and the reason for this discrepancy is unclear [17, 18]. Previous reports describe incidences of close to $100 \%$ whereas we observed writhing behavior in fewer than $50 \%$ of animals $[17,18,23]$. To facilitate comparison with these reports, we used the same definition of writhing as that described by Wadham (1996) and Ambrose $(1998,1999)$ [17, 18, 23].

The proposed cause of writhing behavior is the pain resulting from the alkaline $\mathrm{pH}$ of the $\mathrm{PB}$ solution. The $\mathrm{pH}$ of the solution studied here was 11.02 (measured independently by a commercial compounding pharmacy) within the range $(\mathrm{pH} 10.9-12.6)$ associated with a high incidence of writhing $[17,18,23]$. Current suggestions to alter the effect of $\mathrm{pH}$ focus on changing solution $\mathrm{pH}$ through buffering or the addition of lidocaine to provide analgesia [3, 4]. Wadham [23] reported that buffering a solution of sodium pentobarbital from an original $\mathrm{pH}$ of 12.6 to 9.4 resulted in precipitation [22].

Any study combining behavioral observation in the presence of drugs with sedative properties is inherently confounded by a reduced ability to express behaviors as sedation occurs. This is a limitation of the study design. The use of a vehicle control would address this, but one was not readily available as there were restrictions in obtaining formulation information from the manufacturer of PB. The dose we used in the LL group $(200 \mathrm{mg} / \mathrm{kg})$, was higher than that of Ambrose [17, 18] $(150 \mathrm{mg} / \mathrm{kg})$ and selected based on our institutional standard operating procedure. This may have contributed to the lower incidence of writhing observed by shortening the time after injection when writhing behavior could be expressed, before sedation occurred.

A lack of habituation to handling may have contributed to our findings. The rats used in this study received little or no handling prior to the experiment. Therefore, the stress associated with handling, injection and the observation chamber may have led to a suppression of normal behaviors. The choice to use Sprague-Dawley rats was based on local availability, through an institutional program to make available unused rats for research. As other strains were not studied it is impossible to account for strain differences in drug pharmacokinetics or pharmacodynamics.

Euthanasia can be performed or facilitated by inducing general anesthesia before a physical euthanasia method with drug combinations other than $\mathrm{PB}$, such as xylazineketamine or ethanol (in mice) $[25,26]$. The general principles discussed here, of ensuring that LORR and death occur within an acceptable time following injection, should be applied. It is likely that guidelines for identifying misinjection would need to be determined for each drug or drug combination, accounting for differences in speed of onset of action and differences in effect. Data available for intraperitoneal injection of $70 \%$ or $100 \%$ ethanol in mice suggests 45-60s (75th percentile) to achieve LORR and 3.0-4.25 min for apnea [25]. In contrast, the time to LORR is considerably longer with xylazine-ketamine, taking $6.8 \mathrm{~min}$ for LORR and $8.2 \mathrm{~min}$ for loss of the pedal withdrawal reflex (mean +2 SD for both) [26].

\section{Conclusions}

By coupling the effects of volume and dose with the incidence of misinjections we have suggested practical guidelines to refine overdose with IP sodium pentobarbital as a killing method in rats. Of the groups studied, IP pentobarbital at a dose of $800 \mathrm{mg} / \mathrm{kg}$ (HH group) was the most effective, resulting in the fastest time to death with least variability.

\section{Abbreviations \\ AVMA: American Veterinary Medical Association; CCAC: Canadian Council on Animal Care; CHB: Cessation of heartbeat; CV: Coefficient of variation; $\mathrm{HH}$ : High- dose high-volume; IP: Intraperitoneal; LH: Low-dose high-volume; LL: Low-dose low-volume; LORR: Loss of righting reflex; PB: Sodium pentobarbital}

\section{Acknowledgements}

Not applicable.

\section{Funding}

This study was performed as a University of Calgary Faculty of Veterinary Medicine-Distributed Veterinary Learning Community internship project, funded by the Office of Community Partnerships of the Faculty of Veterinary Medicine and a Canadian Association of Laboratory Animal Science and Canadian Association of Laboratory Animal Medicine Research Fund. JR received an Undergraduate Student Research Award from the Natural Sciences and Engineering Research Council of Canada (NSERC) and DP holds an NSERC Discovery Grant. 


\section{Availability of data and materials}

The datasets generated and analysed during the current study are available in the Harvard Dataverse repository: Pang, Daniel, 2016, "rat IP PB dataset", doi:10.7910/ DVN/PMGCHG, Harvard Dataverse, V1 [UNF:6:A8TqlyFIJXya5kXJ5eWOkQ==]

\section{Authors' contributions}

KZ contributed to study design, collected and analyzed data and wrote the first draft of the paper. CGK collected and analyzed data and revised the manuscript. JR analyzed data and revised the manuscript. DSJP conceived the study, contributed to study design, collected and analyzed data and revised the manuscript. All authors read and approved the final manuscript.

\section{Competing interests}

The authors declare that they have no competing interests.

\section{Consent for publication}

Not applicable.

\section{Ethics approval}

The animal care and use protocol was approved by the Health Sciences Animal Care Committee of the University of Calgary (AC11-0044), in accordance with the guidelines of the Canadian Council on Animal Care.

\section{Author details}

${ }^{1}$ Cornell University College of Veterinary Medicine, Ithaca, NY, USA.

${ }^{2}$ Veterinary Clinical and Diagnostic Sciences, Faculty of Veterinary Medicine, University of Calgary, 3280 Hospital Dr NW, T2N 4 Z6 Alberta, Canada. ${ }^{3}$ Départment de sciences cliniques, Faculté de medicine vétérinaire, Université de Montréal, Saint-Hyacinthe, Québec, Canada. ${ }^{4}$ Groupe de recherche en pharmacologie animale du Québec (GREPAQ), Saint-Hyacinthe, Québec, Canada.

Received: 19 October 2016 Accepted: 18 February 2017 Published online: 21 February 2017

\section{References}

1. Sixth Report from the Commission to the Council and the European Parliament on the Statistics on the number of animals used for experimental and other scientific purposes in the member states of the European Union COM(2010) 511/final 2 [http://ec.europa.eu/environment/ chemicals/lab_animals/reports_en.htm] Accessed: 10 Oct 2016.

2. CCAC Animal Report Data 2014 [http://www.ccac.ca/en_/facts-and-figures/ animal-data] Accessed: 10 Oct 2016.

3. AVMA guidelines for the euthanasia of animals: 2013 edition [https://www avma.org/KB/Policies/Documents/euthanasia.pdf] 15 June 2016.

4. CCAC guidelines on: euthanasia of animals used in science. [http://www. ccac.ca/en_/standards/guidelines] 15 June 2016.

5. Close B, Banister K, Baumans V, Bernoth E-M, Bromage N, Bunyan J, Erhardt W, Flecknell P, Gregory N, Hackbarth H, Morton D, Warwick C. Recommendations for euthanasia of experimental animals: Part 1. Lab Anim. 1996;30:293-316.

6. Demers G, Griffin G, De Vroey G, Haywood JR, Zurlo J, Bédard M. Harmonization of animal care and use guidance. Science. 2006;312:700-1.

7. Chisholm J, De Rantere D, Fernandez NJ, Krajacic A, Pang DS. Carbon dioxide, but not isoflurane, elicits ultrasonic vocalizations in female rats. Lab Anim. 2013;47:324-7

8. Chisholm JM, Pang DS. Assessment of Carbon Dioxide, Carbon Dioxide/ Oxygen, Isoflurane and Pentobarbital Killing Methods in Adult Female Sprague-Dawley Rats. PLoS One. 2016;11, e0162639.

9. Anton F, Peppel P, Euchner I, Handwerker HO. Controlled noxious chemical stimulation: responses of rat trigeminal brainstem neurones to $\mathrm{CO} 2$ pulses applied to the nasal mucosa. Neurosci Lett. 1991;123:208-11.

10. Anton F, Euchner I, Handwerker HO. Psychophysical examination of pain induced by defined CO2 pulses applied to the nasal mucosa. Pain. 1992:49:53-60.

11. Danneman PJ, Stein S, Walshaw SO. Humane and practical implications of using carbon dioxide mixed with oxygen for anesthesia or euthanasia of rats. Lab Anim Sci. 1997;47:376-85.

12. Leach MC, Bowell VA, Allan TF, Morton DB. Aversion to gaseous euthanasia agents in rats and mice. Comp Med. 2002;52:249-57.

13. Niel L, Weary DM. Rats avoid exposure to carbon dioxide and argon. Appl Anim Behav Sci. 2007;107:100-9.
14. Niel L, Stewart SA, Weary DM. Effect of flow rate on aversion to gradual-fill carbon dioxide exposure in rats. Appl Anim Behav Sci. 2008;109:77-84.

15. Peppel $P$, Anton F. Responses of rat medullary dorsal horn neurons following intranasal noxious chemical stimulation: effects of stimulus intensity, duration, and interstimulus interval. J Neurophysiol. 1993;70:2260-75.

16. Thurauf N, Friedel I, Hummel C, Kobal G. The mucosal potential elicited by noxious chemical stimuli with $\mathrm{CO} 2$ in rats: is it a peripheral nociceptive event? Neurosci Lett. 1991;128:297-300.

17. Ambrose N. Refinement of routine procedures on laboratory rodents. PhD Thesis. University of Birmingham; 1998.

18. Ambrose N, Wadham J, Morton D. Refinement of euthanasia. 3rd World Congress on Alternatives and Animal Use in the Life Sciences 1999. p. 1159-1170.

19. Ballard T. Intraperitoneal route of administration - how accurate is this technique. Anim Technol Welf. 2009:8:17-8.

20. Coria-Avila GA, Gavrila AM, Menard S, Ismail N, Pfaus JG. Cecum location in rats and the implications for intraperitoneal injections. Lab Anim (NY). 2007; 36:25-30.

21. Lewis RE, Kunz AL, Bell RE. Error of intraperitoneal injections in rats. Lab Anim Care. 1966:16:505-9.

22. Svendsen O, Kok L, Lauritzen B. Nociception after intraperitoneal injection of a sodium pentobarbitone formulation with and without lidocaine in rats quantified by expression of neuronal c-fos in the spinal cord-a preliminary study. Lab Anim. 2007:41:197-203.

23. Wadham JJB. Recognition and reduction of adverse effects in research on rodents. PhD Thesis. University of Birmingham; 1996

24. Franks NP. General anaesthesia: from molecular targets to neuronal pathways of sleep and arousal. Nat Rev Neurosci. 2008:9:370-86.

25. Allen-Worthington KH, Brice AK, Marx JO, Hankenson FC. Intraperitoneal injection of ethanol for the euthanasia of laboratory mice (Mus musculus) and rats (Rattus norvegicus). J Am Assoc Lab Anim Sci. 2015;54:769-78.

26. Wellington D, Mikaelian I, Singer L. Comparison of ketamine-xylazine and keatmine-dexmedetomidine anesthesia and intraperitoneal tolerance in rats. J Am Assoc Lab Anim Sci. 2013;52:481-7.

\section{Submit your next manuscript to BioMed Central and we will help you at every step:}

- We accept pre-submission inquiries

- Our selector tool helps you to find the most relevant journal

- We provide round the clock customer support

- Convenient online submission

- Thorough peer review

- Inclusion in PubMed and all major indexing services

- Maximum visibility for your research

Submit your manuscript at www.biomedcentral.com/submit
Biomed Central 